\title{
Delayed diagnosis of maternal and congenital syphilis: An unrecognized epidemic?
}

\author{
Zachary Dionisopoulos ${ }^{1}$, Fatima Kakkar², Ana C Blanchard*
}

\begin{abstract}
Syphilis is an infection caused by Treponema pallidum spirochetes. The diagnosis of this sexually transmitted disease may be missed, partly due to the painless nature of genital ulcers in its primary stage. Women in Canada are screened for syphilis in their first trimester of pregnancy, but late pregnancy testing is not done in all provinces to date; therefore, undetected vertical transmission of syphilis may occur. This case emphasizes the importance of recognizing congenital syphilis in infants and young children with unexplained growth problems and biochemical and hematological abnormalities. Congenital syphilis remains a rare diagnosis, but in the context of increased syphilis rates in Canada during recent years, clinicians should consider this diagnosis in infants presenting with compatible clinical manifestations.
\end{abstract}

Suggested citation: Dionisopoulos Z, Kakkar F, Blanchard AC. Delayed diagnosis of maternal and congenital syphilis: An unrecognized epidemic? Can Commun Dis Rep 2022;48(2/3):115-8.

https://doi.org/10.14745/ccdr.v48i23a10

Keywords: syphilis, sexually transmitted infections, pregnancy, congenital infection, epidemiology
This work is licensed under a Creative Commons Attribution 4.0 Internationa License.

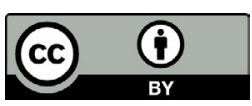

\begin{abstract}
Affiliations
1 Paediatrics Residency Program, Department of Paediatrics, CHU Sainte-Justine, Université de Montréal, Montréal, QC

2 Division of Infectious Diseases, Department of Paediatrics, CHU Sainte-Justine, Université de Montréal, Montréal, QC
\end{abstract}

*Correspondence:

ana.blanchard@umontreal.ca

\section{Introduction}

Congenital syphilis can be challenging to diagnose, as it may mimic other conditions. In addition, clinicians may not consider congenital syphilis due to the rarity of this diagnosis and because women are screened for syphilis in first trimester of pregnancy. Here, we report the case of an infant with intrauterine growth restriction (IUGR) and known congenital cytomegalovirus infection who presented with persistent anemia, thrombocytopenia and liver enzyme elevation. This case highlights the need to have a high index of suspicion in infants presenting with these findings and raises important questions about the need for third trimester screening in pregnant women in Canada.

\section{Case}

A two-month-old infant was admitted to hospital for evaluation of bicytopenia and hepatitis in September 2021. He was born to a G1P1 21-year-old-mother. In the first trimester, she had negative HIV, hepatitis $B$ and $C$ virus and syphilis serologies. She was rubella immune and had negative chlamydia and gonorrhea urine screens. The pregnancy was complicated by oligohydramnios and IUGR in the third trimester. The baby was born prematurely at $35+1 / 7$ weeks of gestation by vaginal delivery, with Apgar scores of 9-10-10. Birth parameters confirmed IUGR with a birth weight of $1,910 \mathrm{~kg}$ (z score -1.48, centile 6.97), a length of $46 \mathrm{~cm}$ (z score -0.09 , centile 46.47$)$ and a head circumference of $31 \mathrm{~cm}$ (z score -0.86, centile 19.53). As part of his IUGR workup, salivary and urine cytomegalovirus $(\mathrm{CMV})$ polymerase chain reaction (PCR) tests were done at day zero and day five of life and were found to be positive at $33,884,416$ and $3,801,894$ copies/mL respectively. The infant had a normal complete blood count and liver enzyme panel at that time. A transfontanellar ultrasound showed small colloidal parenchymal cysts in the right caudate nucleus of unknown origin, without intracranial calcifications. A cerebral magnetic resonance imaging was normal. Ophthalmological and audiological exams were normal. Valganciclovir was initiated at a dose of $16 \mathrm{mg} / \mathrm{kg} /$ dose twice daily, with a plan to complete six months of therapy. He was discharged home at $38+4 / 7$ weeks.

On follow-up at six weeks of age, the infant was found to have a hemoglobin level of $66 \mathrm{~g} / \mathrm{L}$, a platelet count of $77 \times 10^{9} / \mathrm{L}$ and a white blood count of $10 \times 10^{\%} / \mathrm{L}$. His alanine aminotransferase level was $253 \mathrm{U} / \mathrm{L}$, aspartate aminotransferase was $254 \mathrm{U} / \mathrm{L}$, gamma-glutamyl transferase was $256 \mathrm{U} / \mathrm{L}$, alkaline phosphatase level was $690 \mathrm{U} / \mathrm{L}$, albumin was $29 \mathrm{~g} / \mathrm{L}$ and his coagulogram was normal. The anemia and liver enzyme abnormalities were thought to be due to valganciclovir toxicity so treatment was held. He was seen in the clinic a week later without improvement of his blood work findings; therefore, he was admitted to the Paediatrics ward for further investigation. 
On admission, he was afebrile and well overall. On physical exam, he had hepatosplenomegaly and mild diffuse desquamation. He was irritable, but consolable. An extensive workup was done including blood and urine cultures, nasopharyngeal multiplex viral PCR, Epstein Barr virus, parvovirus B19, adenovirus, herpes simplex virus and CMV blood PCRsall results were negative. He required a packed red blood cell transfusion as his hemoglobin had dropped to $57 \mathrm{~g} / \mathrm{L}$.

After 10 days in hospital, the infant developed a new onset low grade fever. Given the persistent unexplained anemia, a bone marrow aspirate was planned. On repeat questioning of the mother alone for possible infectious symptoms, she described for the first-time flu-like symptoms following delivery, as well as a sore throat and genital lesions. In addition, she presented at the time of repeat questioning a bilateral erythematous scaling papular palmar rash (Figure 1) on physical examination, which had appeared in the previous three weeks. Syphilis serologies were immediately ordered on the patient and his mother. They both had strongly-positive syphilis enzyme immunoassays and rapid plasma reagin of 1:64. A lumbar puncture was performed on the infant, with the cerebrospinal fluid analysis showing normal white and red blood cell counts, glucose and protein profiles as well as a negative Venereal Disease Research Laboratory test. A cerebral magnetic resonance imaging was repeated and was normal. A skeletal survey was not conclusive for congenital syphilis, but slightly enhanced periosteal reactions were noted along both femurs and the right tibia (Figure 2).

Placental examination showed only one area of villitis of unknown significance, and no spirochetes were seen on immunochemistry stains.

Figure 1: Rash, woman with secondary syphilis, Montréal, 2021

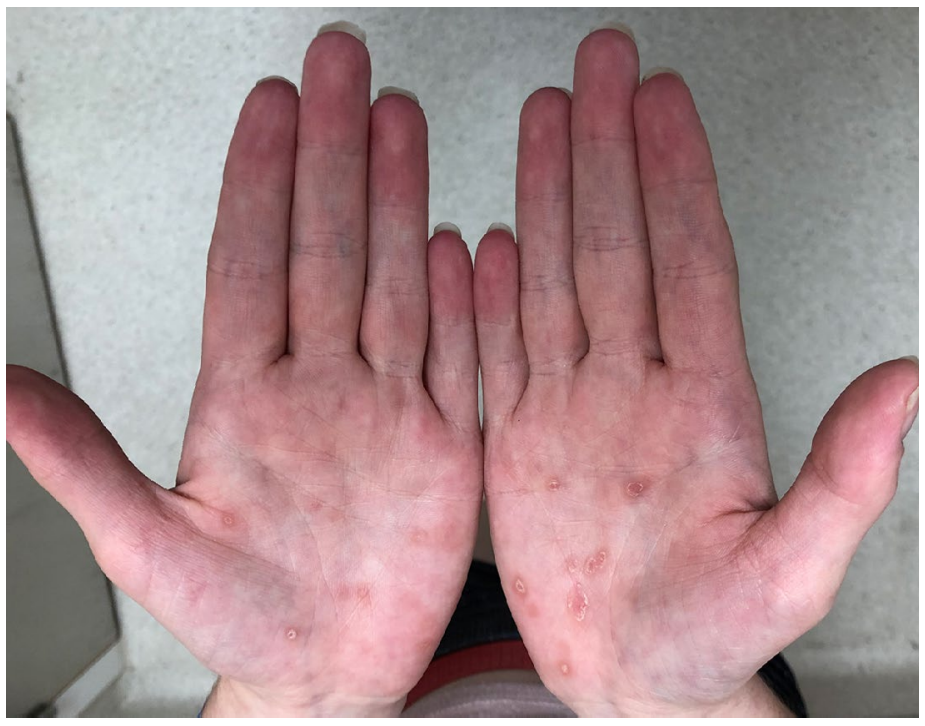

Figure 2: Radiograph of the right femur ${ }^{\mathrm{a}}$ of an infant with congenital syphilis, Montréal, 2021

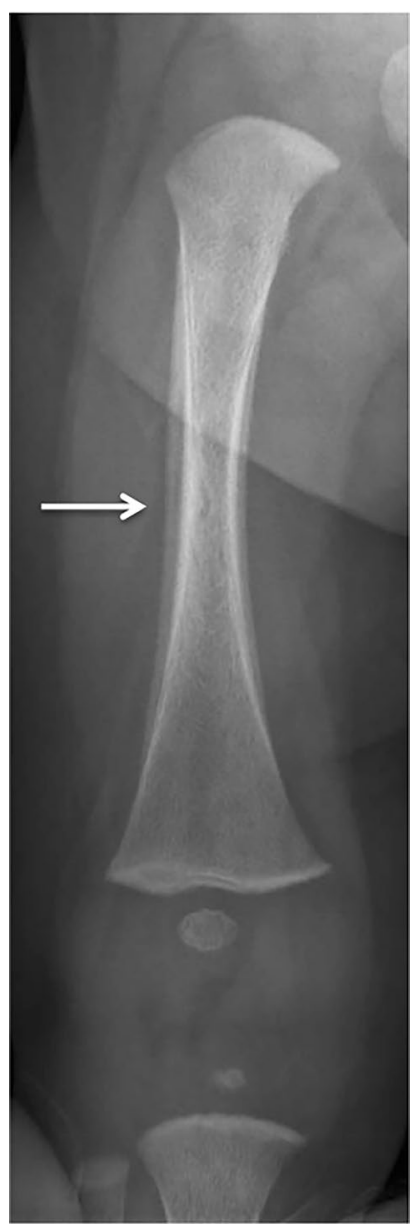

a Slightly enhanced periosteal reactions were noted along both femurs and the right tibia. The right femur is shown here

The infant was treated with a 10-day course of intravenous penicillin $G$, with rapid resolution of his fever and his bloodwork abnormalities. A repeat rapid plasma reagin at the end of treatment was 1:32. The patient was discharged with follow-up in the Congenital Infection Clinic. His mother was referred to an adult infectious diseases clinic, where she received appropriate treatment and serological follow-up.

\section{Discussion}

The rate of syphilis in Canada increased by $85.6 \%$, from 5.0 to 9.3 cases per 100,000 population, from 2010 to 2015. Although this rate was higher in males than in females and was thought to be largely attributable to men who have sex with men, there was a $27.8 \%$ increase among females with individuals aged 20-39 years having the highest rates (1). In a recent report, national rates of syphilis increased by $124 \%$ between 2016 and 2020 , with the largest increase occurring among females (740\%). Nearly a third of cases occurred in women (versus only $8 \%$ in 2016) (2). In addition, the incidence of congenital syphilis has been rising in recent years in Canada, with cases increasing from 
such as that led by the Canadian Paediatric Surveillance Program, are useful to characterize the epidemiology and severity of congenital syphilis which is potentially underdiagnosed in Canadian youth. With the current resurgence of syphilis in Canada, third trimester testing of pregnant women may be considered by public health policy makers in different provinces and territories.

\section{Authors' statement}

All authors were involved in the management of the patient: ZD and ACB made the diagnosis and provided initial management and counselling And FK provided long-term clinical follow-up and management. ZD wrote the initial draft of the manuscript. ACB oversaw manuscript preparation and revisions. All authors read and approved the final manuscript.

The content and view expressed in this article are those of the authors and do not necessarily reflect those of the Government of Canada.

\section{Competing interests}

None.

\section{Acknowledgements}

The authors would like to thank the mother of the patient, who accepted that this data be shared with the scientific community to raise awareness about syphilis.

\section{Funding}

This work did not receive any specific grant from funding agencies in the public, commercial or not-for-profit sectors.

\section{References}

1. Choudhri Y, Miller J, Sandhu J, Leon A, Aho J. Infectious and congenital syphilis in Canada, 2010-2015. Can Commun Dis Rep 2018;44(2):43-8. DOI PubMed

2. Public Health Agency of Canada. Infectious Syphilis and Congenital Syphilis in Canada, 2020 (infographic). Ottawa (ON): PHAC; (updated 2021). https://www.canada.ca/en/ public-health/services/publications/diseases-conditions/ infectious-syphilis-congenital-syphilis-canada-2020.html

3. Gilmour LS, Best EJ, Duncanson MJ, Wheeler BJ, Sherwood J, Thirkell CE, Walls T. High Incidence of Congenital Syphilis in New Zealand: A New Zealand Pediatric Surveillance Unit Study. Pediatr Infect Dis J 2022;41(1):66-71. DOI PubMed

syphilis in young infants with unexplained hepatosplenomegaly, hematological and biochemical abnormalities, despite negative first trimester screening during pregnancy. Surveillance initiatives, 
4. Kimball A, Torrone E, Miele K, Bachmann L, Thorpe P, Weinstock H, Bowen V. Missed Opportunities for Prevention of Congenital Syphilis - United States, 2018. MMWR Morb Mortal Wkly Rep 2020;69(22):661-5. DOI PubMed

5. Canadian Paediatric Surveillance Program. Protocols. Congenital Syphilis. https://cpsp.cps.ca/uploads/studies/ Protocol-congenital-syphilis.pdf

6. Finelli L, Berman SM, Koumans EH, Levine WC. Congenital syphilis. Bull World Health Organ 1998;76 Suppl 2:126-8. PubMed
7. Sheffield JS, Sánchez PJ, Morris G, Maberry M, Zeray F, McIntire DD, Wendel GD Jr. Congenital syphilis after maternal treatment for syphilis during pregnancy. Am J Obstet Gynecol 2002;186(3):569-73. DOI PubMed

8. Provincial Health Services Authority (BC). Interim Guideline on Syphilis Screening in Pregnancy. Vancouver, BC: Perinatal Services BC; September 2019. http://www. perinatalservicesbc.ca/Documents/Guidelines-Standards/ Maternal/Guideline-syphilis-screening-in-pregnancy.pdf

9. Arnold SR, Ford-Jones EL. Congenital syphilis: A guide to diagnosis and management. Paediatr Child Health 2000;5(8):463-9. DOl PubMed

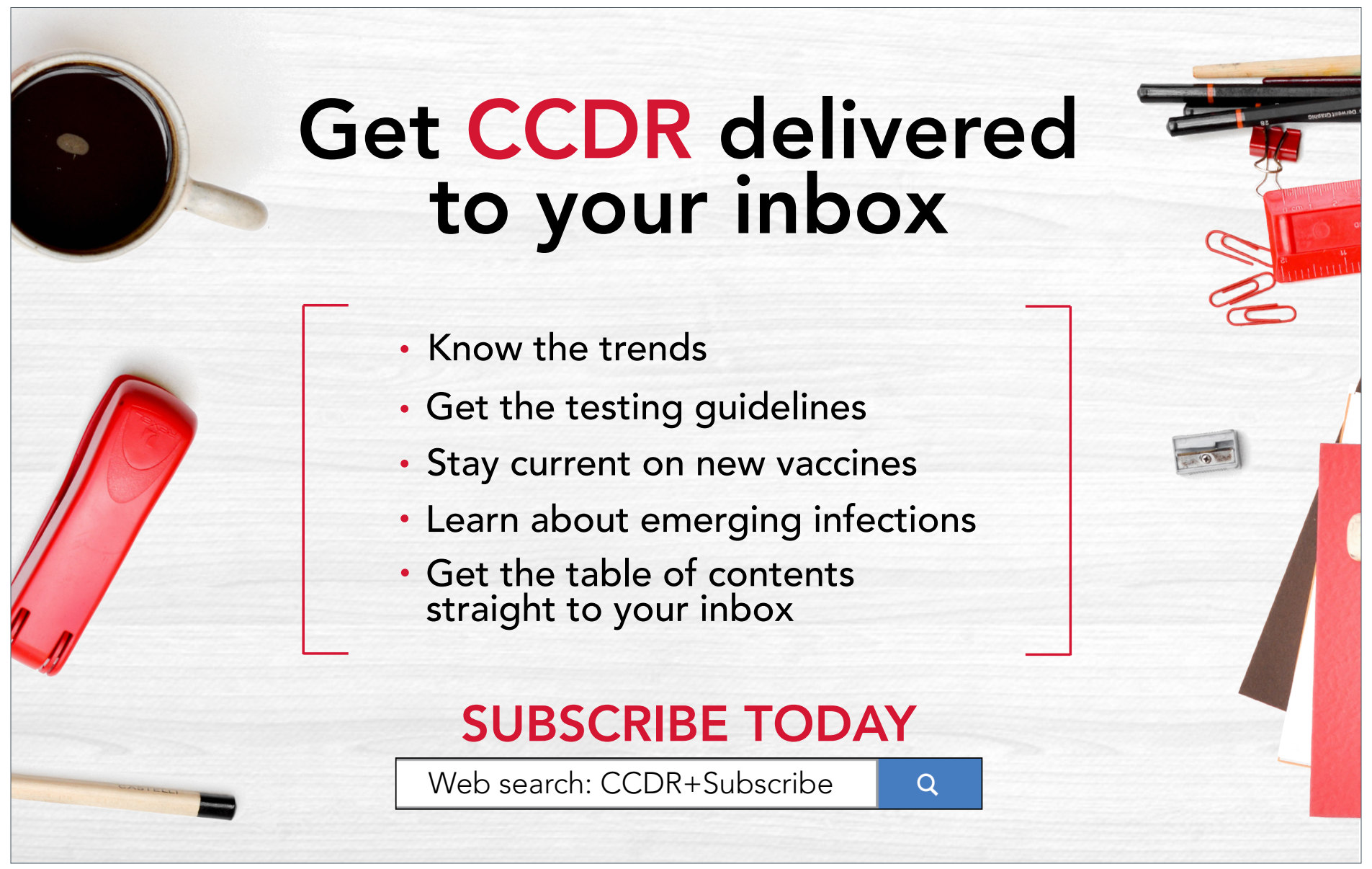

